\title{
碳酸盐耦合同位素 $\left(\Delta_{47}\right)$ 温度计及其在古高度 重建中的应用
}

熊中玉 ${ }^{1,3}, 丁^{1,2^{*}}$, 谢静 $^{1}$

1. 中国科学院青藏高原研究所大陆碰撞与高原隆升重点实验室, 北京 100101;

2. 中国科学院青藏高原地球科学卓越创新中心, 北京 100101 ;

3. 中国科学院大学资源与环境学院, 北京 100049

*联系人, E-mail: dinglin@itpcas.ac.cn

2019-01-14 收稿, 2019-03-09 修回, 2019-03-11 接受, 2019-05-09 网络版发表

青藏高原碰撞造山过程与成矿构造背景科技部深地专项(2016YFC0600303)和国家自然科学基金(41490615)资助

摘要古高度重建为地质模型的建立提供了重要的制约条件, 而古地温的恢复在古高度重建中至关重要. 基于均 质(或同相)同位素交换平衡的碳酸盐耦合同位素温度计 $\left(\Delta_{47}\right.$ 温度计)相比于传统的古温度计具有两个显著的优点: （1）其测量的参数 $\Delta_{47}$ 仅仅与碳酸盐的形成温度相关而不受环境水中 $\delta^{18} \mathrm{O}$ 值影响; (2) $\Delta_{47}$ 的精度能够达到 $0.010 \%$, 换 算到相应的碳酸盐形成温度可优于 $\pm 2^{\circ} \mathrm{C}$. 随着碳酸盐耦合同位素温度计的实验方法的发展, 实现了利用该温度计 精确重建古高度。本文通过总结全球碳酸盐耦合同位素温度计应用于古高度重建中的实例，进一步解析该温度计 在区域抬升历史的恢复以及构建动力学模型中的应用前景.

关键词 碳酸盐耦合同位素, $\Delta_{47}$, 古温度计, 古高度

古高度重建对于解析地球动力学的问题：如造山 带隆升的时间、隆升速率和机制 ${ }^{[1 \sim 3]}$; 地幔动力学 ${ }^{[4,5]}$; 气候变化和季风起源 ${ }^{[6-8]}$ 都有着重要的作用. 现今主要 的古高度计包括: (1) 氧同位素古高度计 ${ }^{[2,9 ~ 11]}$; (2) 氢同 位素古温度-古高度计 ${ }^{[12 ~ 14]}$; (3) 植物叶相-热晗古高度

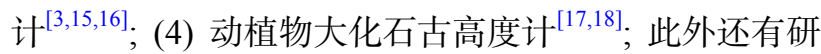
究者运用玄武岩顶底面气泡压差 ${ }^{[19,20]}$ 以及植物孢 粉 ${ }^{[21,22]}$ 来重建古高度.

在以上的古高度计中, 由于很难找到保存完好的 动植物化石、孢粉化石、玄武岩以及火山玻璃剖面, 使得样品相对更容易保存的碳酸盐岩氧同位素古高度 计在古高度重建中应用最为广泛. 利用氧同位素重建 古高度主要基于海洋中的水汽随山脉爬升形成降水的 过程中遵循瑞利分馏原理, 重同位素在降水中富集, 残
留水气团中重同位素亏损，故降水中的氧同位素随着 高度的抬升越来越亏损 ${ }^{[9]}$. 将该原理应用到古高度重建 中则是通过测量碳酸盐岩的 $\delta^{18} \mathrm{O}$ 值 $\left(\delta^{18} \mathrm{O}_{\mathrm{c}}\right)$, 根据碳酸盐水的氧同位素分馏系数与温度的关系反推环境水的 $\delta^{18} \mathrm{O}$ 值, 然后对其进行蒸发量、纬度和季节变化的校 正，得到原始大气降水的 $\delta^{18} \mathrm{O}$ 值 $\left(\delta^{18} \mathrm{O}_{\mathrm{mw}}\right)$, 最后根据模 拟 $\delta^{18} \mathrm{O}_{\mathrm{mw}}$ 与高度的瑞利分馏模型或者两者的经验性模 型, 求得碳酸盐岩沉积时的古高度 ${ }^{[1,2,11,23,24]}$. 由于使用 氧同位素重建古高度, 需要已知碳酸盐岩的 $\delta^{18} \mathrm{O}_{\mathrm{c}}$ 和碳 酸盐岩形成温度反推成岩时环境水的 $\delta^{18} \mathrm{O}$ 值. 以往的研 究中常使用年平均温度来近似取代碳酸盐岩形成温度, 而年平均温度并不能代表碳酸盐岩的实际成岩温度， 不同气候中碳酸盐岩的形成季节不同，使得这些温度 与实际碳酸盐岩形成温度之间的差异达到 $5 \sim 10^{\circ} \mathrm{C}^{[25,26]}$. 
加上重建年均温所存在的误差，造成古高度重建误差 达 \pm 1000 m甚至更大. 此外，还有一些情况无法估计研 究区年均温, 仅能通过碳酸盐岩与现今古土壤的氧同 位素差值来进行古高度恢复 ${ }^{[10]}$.

近年来发展起来的碳酸盐耦合同位素(clumped isotope) 温度计 ${ }^{[27]}$ (亦被翻译为团簇同位素温度计 ${ }^{[28]}$ ), 作为一种热动力学温度计, 可以独立计算碳酸盐的形 成温度, 并且误差可达到 $\pm 2^{\circ} \mathrm{C}$. 通过碳酸盐耦合同位素 温度计, 我们既可直接由碳酸盐岩的 $\delta^{18} \mathrm{O}_{\mathrm{c}}$ 求得 $\delta^{18} \mathrm{O}_{\mathrm{mw}}$, 进而通过瑞利分馏模型或 $\delta^{18} \mathrm{O}_{\mathrm{mw}}$ 与高度的经验关系计 算古高度; 又能利用地表高度/温度梯度计算古高度, 两 种方法相互验证，极大地提高了古高度重建的可信度 和精确度 ${ }^{[29]}$. 此外, 由于碳酸盐耦合同位素温度计仅与 碳酸盐的温度相关，利用其重建的古地温和样品矿物 学特征以及阴极发光特征结合可用于判定碳酸盐岩是 否经历了成岩改造 ${ }^{[25]}$, 解决了使用氧同位素重建古高 度鉴定样品是否保持原始氧同位素信息难的问题.

碳酸盐耦合同位素温度计自身所具备的优点，使 其在地学领域中具有广泛的应用前景，越来越多的实 验室需要建立独立的碳酸盐耦合同位素实验系统. 鉴 于此, 本文的目的主要有两点: 第一是基于碳酸盐耦合 同位素温度计的理论依据，并根据已有的实验测量方 法和实验室转换方程，阐释该温度计在实际应用中的 可行性．第二是通过介绍碳酸盐耦合同位素温度计重 建古高度的原理，分析该温度计在古高度重建中的实 例来阐述碳酸盐耦合同位素温度计在区域抬升历史恢 复和地球动力学机制建立中的作用和应用前景，促进 该方法在国内的发展和推广。

\section{1 基本原理}

碳酸盐耦合同位素温度计的度量参数 $\Delta_{47}$ 定义为, 碳酸盐酸解生成的 $\mathrm{CO}_{2}$ 分子中质量数为 47 的分子丰度 相对于随机状态下该分子丰度的差异程度，故碳酸盐 耦合同位素温度计也称作 $\Delta_{47}$ 温度计 ${ }^{[30,31]}$ ，表达式如下 所示:

$$
\Delta_{47}=\left[\left(\frac{R_{47}}{R_{47}^{*}}-1\right)-\left(\frac{R_{46}}{R_{46}^{*}}-1\right)-\left(\frac{R_{45}}{R_{45}^{*}}-1\right)\right],
$$

$R_{47}, R_{46}, R_{45}$ 分别是测量样品中质量数为 $47,46,45$ 分子 相对于质量数 44 分子的丰度比值, $R_{47}^{*}, R_{46}^{*}, R_{45}^{*}$ 则是随机 分布状态下的对应比值. $\mathrm{CO}_{2}$ 分子同位素体(isotopologues)在随机状态下的丰度如图1所示, 质量数为 47 的分

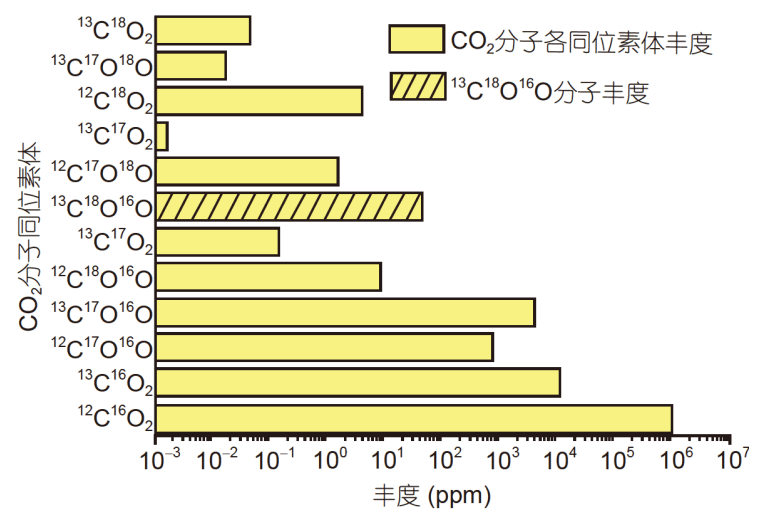

图 1 (网络版彩色) $\mathrm{CO}_{2}$ 各同位素体相对丰度 ${ }^{[32]}$

Figure 1 (Color online) Relative abundance of isotopologues of $\mathrm{CO}_{2}{ }^{[32]}$

子中 ${ }^{13} \mathrm{C}^{16} \mathrm{O}^{18} \mathrm{O}$ 分子的丰度为 $44.4 \mathrm{ppm}(1 \mathrm{ppm}=$ $0.0001 \%)$, 占质量数为 47 的同位素体含量的 $97 \%$, 是 $\Delta_{47}$ 温度计得以应用的关键.

对 $\mathrm{CO}_{2}$ 分子的同位素交换反应： ${ }^{16} \mathrm{O}^{12} \mathrm{C}^{16} \mathrm{O}+$ ${ }^{16} \mathrm{O}^{13} \mathrm{C}^{18} \mathrm{O} \rightleftharpoons{ }^{16} \mathrm{O}^{13} \mathrm{C}^{16} \mathrm{O}+{ }^{16} \mathrm{O}^{12} \mathrm{C}^{18} \mathrm{O}$. 根据统计力学和量子 力学原理, 上述反应的平衡常数 $K$ 是温度的函数, 物质 浓度为 $A$, 理想随机分布状态下的平衡常数为 $K^{*}$, 物质 浓度为 $A^{*}$, 两者取比值得到:

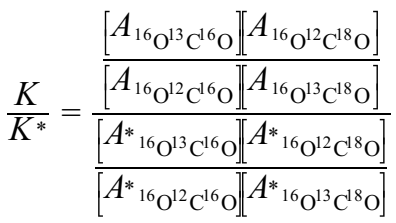

$$
\begin{aligned}
& =\frac{\frac{\left[R^{16} \mathrm{O}^{13} \mathrm{C}^{16} \mathrm{O}\right]\left[R^{16} \mathrm{O}^{12} \mathrm{C}^{18} \mathrm{O}\right]}{\left[R^{16} \mathrm{O}^{12} \mathrm{C}^{16} \mathrm{O} \mathrm{O}\right]\left[R^{16} \mathrm{O}^{13} \mathrm{C}^{18} \mathrm{O}\right]}}{\frac{\left[R^{*}{ }^{16} \mathrm{O}^{13} \mathrm{C}^{16} \mathrm{O}\right]\left[R^{*}{ }^{16} \mathrm{O}^{12} \mathrm{C}^{18} \mathrm{O}\right]}{\left[R^{*}{ }^{16} \mathrm{O}^{12} \mathrm{C}^{16} \mathrm{O}\right]\left[R^{*}{ }^{* 6} \mathrm{O}^{13} \mathrm{C}^{18} \mathrm{O}\right]}} \\
& =\frac{\left(\frac{\Delta{ }^{16} \mathrm{O}^{13} \mathrm{C}^{16} \mathrm{O}}{1000}+1\right)\left(\frac{\Delta^{16} \mathrm{O}^{12} \mathrm{C}^{18} \mathrm{O}}{1000}+1\right)}{\left(\frac{\Delta{ }^{16} \mathrm{O}^{12} \mathrm{C}^{16} \mathrm{O}}{1000}+1\right)\left(\frac{\Delta^{16} \mathrm{O}^{13} \mathrm{C}^{18} \mathrm{O}}{1000}+1\right)} .
\end{aligned}
$$

两边取对数得到:

$$
-1000 \ln \left(\frac{K}{K^{*}}\right)=\Delta{ }^{16} \mathrm{O}^{13} \mathrm{C}^{18} \mathrm{O}-\Delta{ }^{16} \mathrm{O}^{12} \mathrm{C}^{18} \mathrm{O}-\Delta{ }^{16} \mathrm{O}^{13} \mathrm{C}^{16} \mathrm{O} .
$$

根据平衡常数与温度之间的关系，导出 $\Delta_{47}$ 与 $1 / T^{2}$ 之间存在近似线性关系，奠定了 $\Delta_{47}$ 温度计用于古地温 重建的理论基础 ${ }^{[33]}$. 由于 $\Delta_{4}$ 温度计的 $\mathrm{CO}_{2}$ 分子由碳酸 盐酸解释放，因而在酸解的过程中存在动力学分馏使 得碳酸盐酸解平衡得到的 $\mathrm{CO}_{2}$ 分子和理想 $\mathrm{CO}_{2}$ 分子之 
间存在差异 ${ }^{[34]}$ (图S1).

在实际应用中，实验室通过气体同位素质谱仪测 量 $\mathrm{CO}_{2}$ 分子中的 $R_{45} \sim R_{47}$ 计算得到 ${ }^{13} \mathrm{C},{ }^{18} \mathrm{C}$ 和 ${ }^{17} \mathrm{C}$ 的同位 素比值 $R_{13}, R_{18}$ 以及 $R_{17}$, 并将其转换为同位素丰度计算 随机状态下质量数为 $45 \sim 47$ 的同位素比值 $R_{45} \sim R_{47}^{*}$, 表达 式(1)可进一步导出为

$$
\begin{aligned}
\Delta_{47}= & \left(\frac{R_{47}}{2 R_{13} \times R_{18}+2 R_{17} \times R_{18}+R_{13} \times\left(R_{17}\right)^{2}}\right. \\
& \left.-\frac{R_{46}}{2 R_{18}+2 R_{13} \times R_{17}+\left(R_{17}\right)^{2}}-\frac{R_{45}}{R_{13}+2 R_{17}}+1\right) \\
& \times 1000 .
\end{aligned}
$$

需要指出的是选择不同的计算参数造成的差异可 达到 $0.05 \% 0{ }^{[35,36]}$, 因此不同的实验室之间尽量选择一致 的计算参数便于实验室之间数据对比. 此外, 根据相似 原理可推算 $\Delta_{48}$ 和 $\Delta_{49}$, 分别用于检测样品是否纯化干净 (如去除了烃类化合物以及硫化物的干扰) ${ }^{[37]}$ 和背景值 校正 ${ }^{[38,39]}$.

\section{2 实验方法}

\section{1 样品的制备和前处理}

$\Delta_{47}$ 温度计测量的碳酸盐样品分无机成因和生物成
因. 无机成因样品主要指合成方解石、古土壤中的钲 质结核、钙华和石灰华等. 合成方解石可通过在已知 温度下将饱和的 $\mathrm{NaHCO}_{3}$ 与 $\mathrm{CaCl}_{2}$ 混合之后放在空气中 被动去气(高温条件下需要加压避免沸腾)沉淀方解 石 $^{[40 \sim 42]}$; 或者是在饱和 $\mathrm{CaCO}_{3}$ 溶液中通人氮气主动去 气生成方解石 ${ }^{[32,43]}$. 然后取出合成的方解石进行干燥 后研磨均匀以备用(图S2). 其他样品(古土壤、钙华和 石灰华)则需要使用微型牙钻或者研磨工具取得小于 200 目的粉末以备用. 生物碳酸盐需要先经过超声清洁 并用过氧化氢溶液清洗以除去其中的有机质，然后钻 取或研磨成小于 200 目的粉末备用. 所有样品都可通过 $\mathrm{X}$-射线衍射或者扫描电子显微镜鉴定其矿物特征，并 由此选用不同的酸解分馏系数 ${ }^{[44-46]}$.

\section{2 样品的酸解、 $\mathrm{CO}_{2}$ 的纯化以及测量}

现今对碳酸盐进行酸解以及对该反应产生的 $\mathrm{CO}_{2}$ 气体的纯化系统主要分为两类: 一类是基于微量样品 $(0.15 \sim 0.2 \mathrm{mg})$ 而建立起来的改进版Keil IV装置, 该装 置在 $70{ }^{\circ} \mathrm{C}$ 下使用浓度为 $103 \% \sim 105 \%$ 的磷酸将碳酸盐酸 解产生的 $\mathrm{CO}_{2}$ 气体通过微型液氮冷阱以及微型填充色 谱柱构成的冷阱纯化后送人气体同位素质谱仪进行测 量 $^{[47-49]}$ (图2(a)). 微量样品碳酸盐纯化装置需要的样品 量少, 应用范围广, 但是精度较低, 需要多次测量以达 (a)

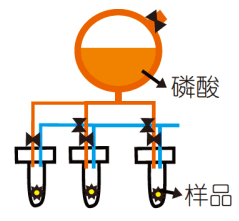

Keil IV 装置酸解

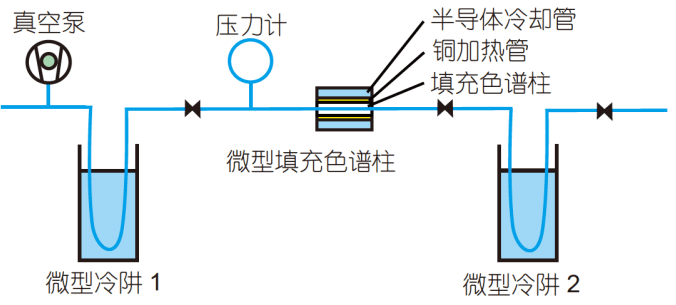

(b)

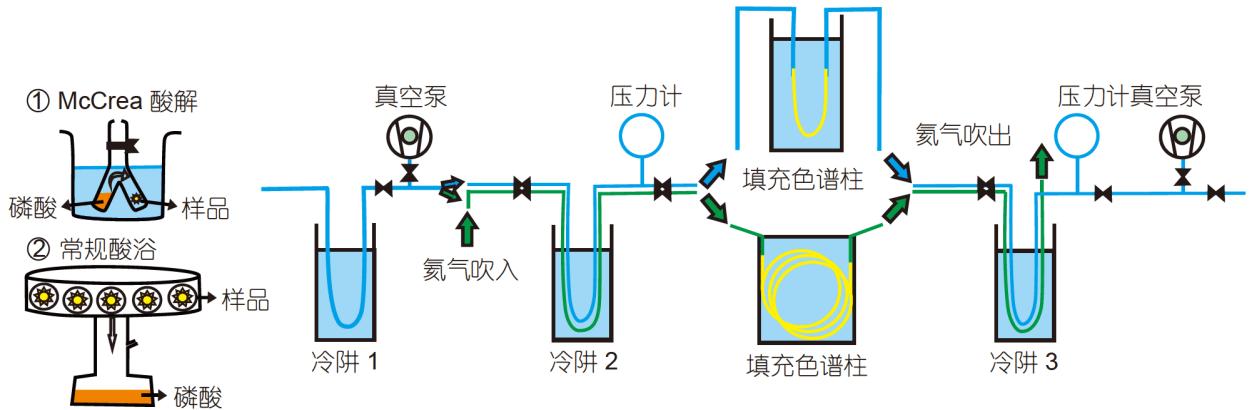

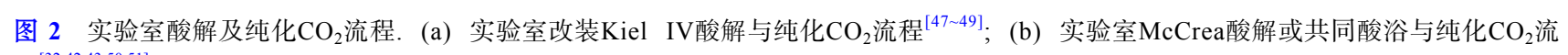
程 $[32,42,43,50,51]$

Figure 2 Laboratory acid digestion and purification of $\mathrm{CO}_{2}$. (a) Acid digestion and purification through Kiel IV device ${ }^{[47-49]}$; (b) acid digestion and purification through McCrea or common bath devices ${ }^{[32,42,43,50,51]}$ 
到测量精度要求. 另一类是制备大样品量 $(5 \sim 15 \mathrm{mg})$ 的 碳酸盐装置(图2(b)), 这些装置包括使用 $\mathrm{McCrea}$ 酸解装 置或者使用配置自动进样器的共同酸浴装置在 $25^{\circ} \mathrm{C}$ $\left(70\right.$ 或 $\left.90{ }^{\circ} \mathrm{C}\right)$ 下使用浓度为 $103 \% \sim 105 \%$ 的磷酸酸解生成 $\mathrm{CO}_{2}$ 气体, 然后利用液氮冷阱将产生的 $\mathrm{CO}_{2}$ 气体提取出 来通人 $-78 \sim-90^{\circ} \mathrm{C}$ 冷阱中去除 $\mathrm{CO}_{2}$ 气体中可能含有的 水; 随后将 $\mathrm{CO}_{2}$ 气体通人保持在 $-15 \sim-20^{\circ} \mathrm{C}$ 条件下的静 态(无载气)或者动态(氦气作为载气)的填充色谱柱中 去除其中可能含有的烃类干扰物; 之后可将 $\mathrm{CO}_{2}$ 气体通 人后续-78 $-90^{\circ} \mathrm{C}$ 的冷阴中再次去水; 最后将纯化后 的 $\mathrm{CO}_{2}$ 手动(或自动)送人气体同位素质谱仪进行测 量 $^{[32,42,43,50,51]}$. 此外, 有研究认为填充色谱柱的温度低于 $-20^{\circ} \mathrm{C}$ 可能会引起明显的同位素分馏, 所以各实验室的 前处理系统应尽量将其温度控制在 $-20^{\circ} \mathrm{C}$ 以上相对稳 定的温度, 以保证实验数据的准确性 ${ }^{[52]}$. 大样品量纯化 系统具有产气量大, 单次测量精度高的优点, 但是对样 品量的要求较大, 很多研究方向的样品量都无法达到.

迄今为止, 几乎所有的 $\mathrm{CO}_{2}$ 气体耦合同位素测定都 在赛默飞世尔公司(Thermo Fisher)出产的气体同位素 质谱仪MAT 253或者253 Plus上完成, 数据测定在双路 进样模式下进行，根据样品量的要求可以采用传统的 大气量样品双路进样系统, 或者针对小气量样品的微 型双路进样系统. MAT 253气体同位素质谱仪中质量 数44 49的法拉第杯电阻配置分别为 $10^{8}, 10^{10}, 10^{11}$, $10^{12}, 10^{12}, 10^{12} \Omega^{[31]}$; 而在 253 Plus气体同位素质谱仪 上, 为了提高质量数 $47 \sim 49$ 的信噪比, 为质量数 $47 \sim 49$ 的 信号接收处配置了 $10^{13} \Omega$ 电阻法拉第杯，并在质量数 47.5 处配备了一个法拉第杯 (电阻为 $10^{13} \Omega$ ) 专门用于检 测质量数 47 的背景值 ${ }^{[49]}$.

\section{3 实验数据的标准化}

在实际应用中，由于不同实验室所使用的仪器和 测量参数各有差异, 所以需要建立统一的标准, 便于不 同实验室之间的数据能够在统一标准框架下进行对比 和分析. 基于上述原因, Dennis等人 ${ }^{[53]}$ 以不同温度下的 平衡气体的 $\Delta_{47}$ 测量值和理论值为基础建立了绝对参考 系(absolute reference frame), 也叫做二氧化碳平衡标尺 (carbon dioxide equilibrium scale), 使得所有实验室都可 以将自己的数据校正到该绝对参考系中进行比较. 而 最近John和Bowen ${ }^{[54]}$ 为了解决 $\Delta_{47}$ 数据处理复杂, 校准 参数多且不一致, 耗时长并且对使用者的数学能力要 求高的问题而设计了一款数据处理软件Easotope, 使用
者只需要将质谱仪测量得到的数据拖拽到软件中, 便 可自动计算出所需各类 $\delta$ 值、 $\Delta_{47}$ 值以及其标准偏差.

绝对参考系建立的过程就是利用制备的平衡气体 对质谱仪的非线性特征和“度量刻度压缩效应”进行校 正的过程 ${ }^{[11,53]}$. 质谱仪的非线性特征的产生是由于质 谱仪中可变体积储样容器压力差别, 进入离子源的 $\mathrm{CO}_{2}$ 产生了不同强度的质量数 44 离子流对质量数 47 的背景 基线产生影响, 致使相同平衡温度气体的 $\delta_{47}$ 值和 $\Delta_{47}$ 值 存在经验性线性关系，因此在实际应用中需要利用该 线性关系对 $\Delta_{47}$ 值进行校正 ${ }^{[31,53]}$. 对于稳定运行的质谱 仪, 其产生的背景干扰相对稳定, 可直接用一定温度下 的平衡气体的 $\delta_{47}$ 值和 $\Delta_{47}$ 值的斜率校正质谱仪的非线 性干扰. 而对于运行不稳定的质谱仪, 质量数 47 背景值 测量出现明显负信号时，平衡气体的 $\delta_{47}$ 值和 $\Delta_{47}$ 值斜率 变化很大, 此时还需进行负背景值校正. 如果质谱仪质 量数44法拉第接收杯的狭缝远远宽于质量数 47 的接收 杯狭缝, 可以通过质量数 44 的信号强度与质量数 47 背 景最小值的对应关系进行校正 ${ }^{[38]}$; 而对于两个质量数 接收杯狭缝差异不大的质谱仪，可以通过质量数 49 的 负信号来校正质量数 47 的负背景值信号 ${ }^{[39]}$. 对于配置 了质量数 47.5法拉第杯的质谱仪 253Plus，可直接测量 47.5 法拉第杯负信号用于负背景值校正 ${ }^{[49]}$. 此外，还可 以利用具有相同形成温度(即具有相同 $\Delta_{47}$ 值)而同位素 组成不同(即 $\delta_{47}$ 值不同)的碳酸盐标样(如ETH-1和ETH2)实现背景值校正 ${ }^{[5]} \cdot \mathrm{CO}_{2}$ 气体进人离子源会发生碎裂 和重排反应，使得质谱测量值和理论计算值(真实值)之 间存在一个比例系数关系，这种现象即“度量刻度压缩 效应, ${ }^{[31,53]}$, 故还需要对某一温度下平衡气体经非线性 校正后的 $\Delta_{47}$ 测量值(平衡气体 $\delta_{47} \Delta_{47}$ 拟合线截距值)和 其相应的理论计算值进行校正得到 $\mathrm{CO}_{2}$ 气体真实值.

绝对参考系建立的具体操作步骤即对不同温度下 的平衡气体(EG)的 $\delta_{47}$ 和 $\Delta_{47}$ 的测量值(相对于实验室工 作气体(WG)测定得到)进行散点作图, 从而获得不同温 度平衡气体(EG)各自拟合曲线(EGL)的共同斜率 $K_{\mathrm{EGL}}$ 和各自截距 $B_{\mathrm{EGL}}$, 然后将不同温度下的 $\Delta_{47(\mathrm{EG}-\mathrm{WG})}$ 和对应 温度下的理论计算值 $\left(\Delta_{47-\mathrm{RF}}\right)$ 进行投点，得到实验室的 经验性转换方程 $(\mathrm{ETF})$ 的斜率 $\left(K_{\mathrm{ETF}}\right)$ 和截距 $\left(C_{\mathrm{ETF}}\right)$.

$$
\begin{aligned}
& \Delta_{47-[\mathrm{EG}-\mathrm{WG}] 0}=K_{\mathrm{EGL}} \times \delta_{47-[\mathrm{EG}-\mathrm{WG}]}+B_{\mathrm{EGL}}, \\
& \Delta_{47-\mathrm{RF}}=K_{\mathrm{ETF}} \times \Delta_{47-[\mathrm{EG}-\mathrm{WG}] 0}+C_{\mathrm{ETF}} .
\end{aligned}
$$

实验室的经验性转换方程建立后，测量未知样品 (SG)时, 样品的 $\Delta_{47-R F}$ 的校正过程如下所示: 


$$
\begin{aligned}
& \Delta_{47-[\mathrm{SG}-\mathrm{WG}] 0}=\Delta_{47-[\mathrm{SG}-\mathrm{WG}]}-\delta_{47-[\mathrm{SG}-\mathrm{WG}]} \times K_{\mathrm{EGL}}, \\
& \Delta_{47-\mathrm{RF}}=K_{\mathrm{ETF}} \times \Delta_{47-[\mathrm{SG}-\mathrm{WG}] 0}+C_{\mathrm{ETF}} .
\end{aligned}
$$

由于离子源内的碎裂和重排反应随着仪器状态变 化而发生改变, $\mathrm{CO}_{2}$ 气体在酸解以及纯化过程中由于实 验条件改变等存在系统性偏差, 使得经验性转换方程 在使用一段时间后会发生变化，所以需要不断地使用 平衡气体来监测经验性转换方程的变化. 而在实际应 用中，各实验室还可以使用国际标样的参考 $\Delta_{47}$ 值和实 验室的测量值进行投点得到二级参考系来监测实验室 的经验性转换方程是否有效. 现今已经具有 $\Delta_{47}$ 发表值, 可供其他实验室参考对比的标样主要有NBS19, CM2, ETH-1, ETH-2, ETH-3, ETH-4, LESVC, 其参考值如表 1所示. 此外，对于在绝对参考系构建之前就已经发表 的数据, 亦可以使用相似的经验性关系构建二级参考 系将数据校正到绝对参考系中 ${ }^{[53]}$.

早期的碳酸盐样品主要在 $25^{\circ} \mathrm{C}$ 条件下酸解, 后期 为提高实验室的制样效率提升了酸解温度, 因此需要 对不同的酸解温度下获得的 $\Delta_{47}$ 数据进行校正. 对于碳 酸盐酸解的校正主要分两种情况讨论, 一种是实验室 的经验性校正, 主要是以 $25^{\circ} \mathrm{C}$ 酸解得到的 $\Delta_{47}$ 值为基准, 该值不经过任何校正, 然后通过在 90 (或70)和 $25^{\circ} \mathrm{C}$ 下酸 解同一碳酸盐矿物, 求得两种酸解温度下 $\Delta_{47}$ 差异值来 进行校正. 以实验室合成方解石为例, 现今 $90^{\circ} \mathrm{C}$ 酸解校 正值存在 $0.081 \%$ ， $0.082 \%$ 以及 $0.092 \%$ 三个校正 值 ${ }^{[51,56,57]} ; 70{ }^{\circ} \mathrm{C}$ 校正值为 $0.062 \% 0{ }^{[57]}$. 而不同的碳酸盐矿 物, 其实验室经验性校正值存在差异 ${ }^{[58]}$. 另一种情况是 Guo等人 ${ }^{[34]}$ 在理论层次上考虑碳酸盐晶格酸解到 $\mathrm{CO}_{2}$ 过程中存在的耦合同位素分馏, 运用晶格动力学模型 计算得出在不同温度下酸解的方解石发生的耦合同位
素分馏方程:

$\Delta_{47-\text { 酸解 }}=0.0242+0.189 \times \frac{10^{5}}{T^{2}}$.

该理论计算模型主要考虑了磷酸酸解过程中碳酸 分解的同位素分馏, 而对于磷酸从碳酸盐矿物中置换 出碳酸的过程和不同碳酸盐矿物的矿物学特征所造成 的分馏差异并未有很好的限制. 由于采用不同的校正 方法得到的 $\Delta_{47}$ 值的差异可高达 $0.023 \%{ }^{[34,57]}$, 因此需要 谨慎选择酸解校正值. 此外酸解校正不能简单地根据 碳酸盐矿物在两个不同酸解温度下得到的截距取得酸 解校正值, 而可能还需要定量评估矿物的种类, 同种矿 物的非均质性以及反应条件(例如: 温度、时间、水汽 含量 $)^{[59 \sim 61]}$.

\section{4 实验室经验性转换方程}

自 Ghosh等人 ${ }^{[32]}$ 发表了 $1 \sim 50^{\circ} \mathrm{C}$ 之间合成的方解石 $\Delta_{47}$ 值和温度的经验性转换方程以来, 越来越多的研究 者在更广阔的温度范围内对两者的经验性关系进行了 研究, 使得利用 $\Delta_{47}$ 值和温度关系进行内插以及外推更 具可行性 ${ }^{[32,40 \sim 43,56,60,62]}$. 现今实验室的经验性转换方程 误差一般在 $0.010 \% 0(1 \sigma)$, 甚至能达到 $0.005 \%$ o $(1 \sigma)$. 然而 随着实验室合成方解石数据的增多, 这些数据得到的 $\Delta_{47}$ 值和温度之间的经验关系却呈现出两种不同的趋 势. 一种是以 Ghosh等人 ${ }^{[32,43,62]}$ 为代表的数据结果得出 了斜率较高的经验性转换曲线; 其他研究者的数据则 呈现出斜率偏小的趋势 ${ }^{[40 ~ 42,56,60]}$. 究其原因除了早期数 据未校正到绝对参考系上之外，还可能是因为各个实 验室在酸解方法, 样品纯化和数据推算上存在差 异 ${ }^{[36,43,56]}$. 本文将所有已发表的数据都校正到绝对参考

表 1 部分标样的 $\Delta_{47}$ 参考值

Table 1 Reference $\Delta_{47}$ values of some reference materials

\begin{tabular}{ccccc}
\hline 碳酸盐标样 & ${ }^{13} \mathrm{C}(\%)$ vs. VPDB & ${ }^{18} \mathrm{O}(\%)$ vs. VPDB & $\Delta 47(\%)$ 绝对标尺 & 文献 \\
\hline Carrara Marble & $2.285 \pm 0.006$ & $-1.765 \pm 0.010$ & $0.395 \pm 0.008$ & $0.392 \pm 0.017$ \\
NBS-19 & $1.995 \pm 0.015$ & $-2.221 \pm 0.016$ & $0.779 \pm 0.008$ & {$[53]$} \\
LSVEC & $-46.476 \pm 0.046$ & $-26.356 \pm 0.044$ & $0.258 \pm 0.003$ & {$[55]$} \\
ETH-1 & $2.00 \pm 0.01$ & $-2.19 \pm 0.02$ & $0.256 \pm 0.004$ & {$[47]$} \\
ETH-2 & $-10.20 \pm 0.01$ & $-18.58 \pm 0.02$ & $0.691 \pm 0.004$ & {$[55]$} \\
ETH-3 & $1.67 \pm 0.01$ & $-1.75 \pm 0.02$ & $0.507 \pm 0.004$ & {$[55]$} \\
ETH-4 & $-10.22 \pm 0.01$ & $-18.67 \pm 0.02$ & & {$[55]$} \\
\hline
\end{tabular}


上, 然后使用统一的酸解分馏校正参数: $0.082 \% 0\left(90^{\circ} \mathrm{C}\right)$, $0.062 \% 0\left(70^{\circ} \mathrm{C}\right)$ 进行校正, 最后利用最小二乘法在 $95 \%$ 的 置信条件下得到复合转换方程(10). 该方程的拟合系数 为 0.964 , 表明该复合转换方程基本可以解决上述两类 经验性转换方程斜率上的不一致(图3(a)).

$$
\begin{aligned}
\Delta_{47}= & (0.0415 \pm 6.116 E-4) \times \frac{10^{6}}{T^{2}} \\
& +(0.2359 \pm 0.0074) .
\end{aligned}
$$

使用同样的方法对生物方解石转换数据进行分析, 结果显示采用统一的酸解系数并不能解决不同生物转 换方程之间存在的差异(图3(b) $)^{[32,46,57,63 \sim 67]}$. 导致该差 异的原因除了酸解方法，样品纯化和数据推算方法的 差异之外, 不同生物迥异的生存环境, 种类差异以及生 命效应等都可能造成转换方程的不一致 ${ }^{[65,66]}$.

此外，还有研究者对白云石 ${ }^{[68,69]}$ 、磷铁矿 ${ }^{[70]}$ 以及 石灰华 ${ }^{[71,72]}$ 等进行了实验室转换, 由于数据较少, 其可 行性还有待更多的转换方程来验证.

\section{5 误差分析}

$\Delta_{47}$ 的误差主要来自于两个方面：样品本身的非均 质性和实验误差. 样品本身的非均质性是由于碳酸盐 的同位素交换反应可能未达到平衡或遭受外界流体和 温度改造使得其同位素组分分布不均匀, 故取样过程中 在不同位置取样测量结果存在差异. 因此, 在选取测量 样品的过程中需要尽量选择均质性较好的样品. 此外, 对样品进行复样分析 $(n \geqslant 3)$ 是减少样品本身误差的关 键 ${ }^{[43,47]} . \Delta_{47}$ 的实验误差来源主要有 4 个方面: (1) 样品制 备和纯化过程中发生的同位素分馏作用, 例如样品在酸 解过程中可能未达到同位素交换平衡以及反应后得到

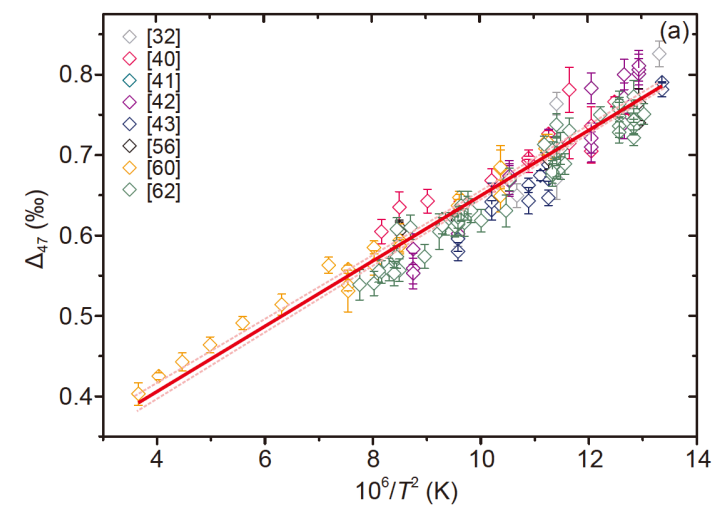

的 $\mathrm{CO}_{2}$ 气体在纯化运移过程中发生同位素分馏 ${ }^{[59]}$; 与质谱仪相关的误差主要有：未加校正的背景值 ${ }^{[31,39]}$; 样品进人离子源发生的碎裂和重排作用引起的“度量刻 度压缩效应”, ${ }^{[31]} ; \mathrm{CO}_{2}$ 与毛细管内壁之间交互发生同位 素交换 ${ }^{[51]} ;$ (3) 不同实验室在计算 $\Delta_{47}$ 选择的计算参数不 一致 ${ }^{[35,36]}$; (4) 经验函数本身所产生的系统性误差 ${ }^{[53]}$.

\section{$3 \Delta_{47}$ 温度计在古高度重建中的应用}

\section{1 原理}

利用碳酸盐耦合同位素进行古高度重建的原理依 托降水中的氧同位素以及温度随着海拔的升高而下降 ${ }^{[9]}$ (图4(a)). 对于初始沉积在不同时代 $\left(t_{1} \sim t_{5}\right)$ 且不同高度的 碳酸盐岩(古土壤结核以及泥晶灰岩)样品, 其古高度重 建研究思路主要有两种: 一种是根据 $\Delta_{47}$ 温度计得到碳 酸盐岩形成温度 $\left(T\left(\Delta_{47}\right)\right)$, 通过碳酸盐-水的氧同位素分 馏系数推算出环境水的氧同位素值，进而估算 $\delta^{18} \mathrm{O}_{\mathrm{mw}}$. 此外，还需利用低海拔靠近海洋地区同期碳酸盐岩的 环境水氧同位素值或古大气降水模型模拟得到的低海 拔处降水氧同位素值作为同期水汽来源的 $\delta^{18} \mathrm{O}_{\mathrm{mw}}$; 将 样品与同期水汽来源的 $\delta^{18} \mathrm{O}_{\mathrm{mw}}$ 进行比较得到两者差值 $\left(\Delta^{18} \mathrm{O}\right)$, 最后利用瑞利分馏模型重建古高度 ${ }^{[73]}($ 图4(b)). 瑞利分馏模型一般适用于水汽来源和气候条件都相对 稳定的低纬地区 (低于 $35^{\circ} \mathrm{N}$ 或 $\left.35^{\circ} \mathrm{S}\right)$, 并且地表抬升是造 成 $\delta^{18} \mathrm{O}_{\mathrm{mw}}$ 下降的主导因素.

另一种是利用 $T\left(\Delta_{47}\right)$ 与高度之间的经验性关系重 建古高度, 具体包括两种途径: 一是恢复不同沉积时期 $\left(\mathrm{t}_{1} \sim \mathrm{t}_{4}\right)$ 与现今高度相当的样品的 $T\left(\Delta_{47}\right)$ 以及 $\delta^{18} \mathrm{O}_{\mathrm{mw}}$, 然后 与现今 $\left(\mathrm{t}_{5}\right)$ 样品形成温度和 $\delta^{18} \mathrm{O}_{\mathrm{mw}}$ 进行相似性对比 ${ }^{[74 \sim 76]}$

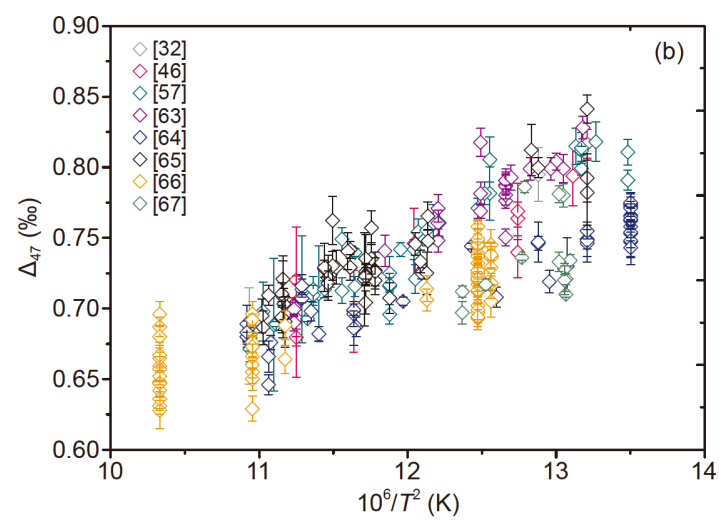

图 3 碳酸盐实验室经验性转换方程. (a) 实验室合成方解石 $\Delta_{47}$ 与温度转换方程 ${ }^{[32,40-43,56,60,62]}$; (b) 生物方解石 $\Delta_{47}$ 与温度转换方程 [32,47,57,63 -67] Figure 3 Laboratory empirical calibration of carbonate. (a) Calibration of synthetic calcite ${ }^{[32,40-43,56,60,62]}$; (b) calibration of bio-calcite ${ }^{[32,46,57,63-67]}$ 

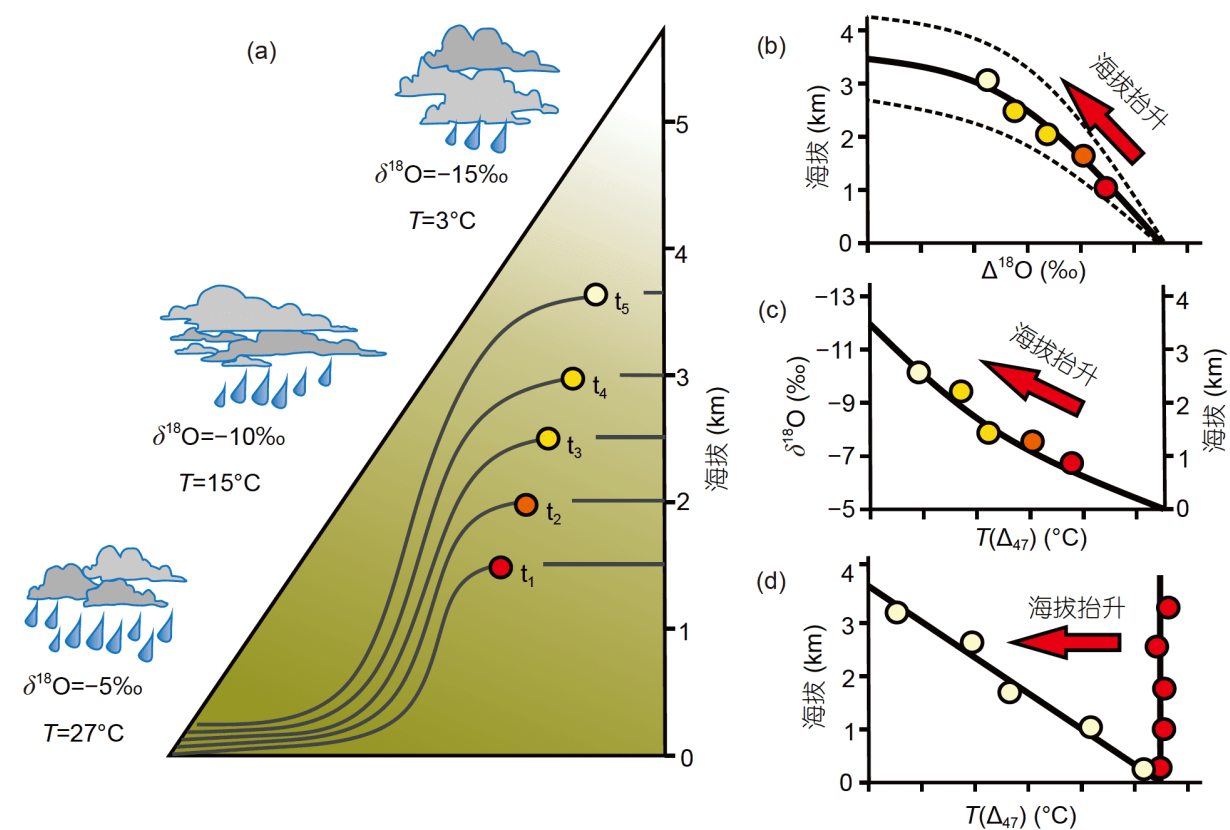

图 $4 \Delta_{4}$ 温度计应用于古高度重建原理. (a) 降水氧同位素和大气温度随着高度的升高而下降 ${ }^{[9]}$; (b) 由 $T\left(\Delta_{47}\right)$ 恢复的 $\delta^{18} \mathrm{O}_{\mathrm{mw}}$ 应用于瑞利分馏模 型重建古高度 ${ }^{[73]}$; (c) 利用不同时期相似高度样品 $T\left(\Delta_{47}\right)$ 和 $\delta^{18} \mathrm{O}_{\mathrm{mw}}$ 与高度相关性重建古高度 ${ }^{[74-76]}$; (d) 利用同期不同高度样品与对应现今样品 $T$ $\left(\Delta_{47}\right)$ 的差异和相应高度差异重建古高度 ${ }^{[77]}$

Figure 4 Theories regarding the application of $T\left(\Delta_{47}\right)$ in paleoelevation reconstruction. (a) $\delta^{18} \mathrm{O}_{\mathrm{mw}}$ and air temperature decrease with the increase of elevation ${ }^{[9]}$; (b) application of Raleigh-fractionation-based model to reconstruct paleoelevation ${ }^{[73]}$; (c) application of the relationship of $T\left(\Delta_{47}\right)$ and $\delta^{18} \mathrm{O}_{\text {mw }}$ with elevation at different time periods to reconstruct paleoelevation ${ }^{[74-76]}$; (d) application of the difference of $T\left(\Delta_{47}\right)$ between ancient and modern samples at several elevations to reconstruct paleoelevation ${ }^{[77]}$

(图4(c)); 其次是恢复同一时期(如 $\mathrm{t}_{1}$ )不同高度样品的 $T$ $\left(\Delta_{47}\right)$, 将其直接和相应高度的现今样品 $\left(\mathrm{t}_{5}\right)$ 的气温直减 率进行比较 ${ }^{[77]}$. 该方法需要将 $T\left(\Delta_{47}\right)$ 校正到现今大气年 平均温上, 从而应用现今气温直减率恢复古高度. 但是 不同地区土壤温度受到太阳辐射、土壤深度以及植被 覆盖的影响不一, 因此不同地区土壤温度和大气年平 均温的相关性也存在差异 ${ }^{[25,26]}$.

\section{2 样品选取和鉴定}

$\Delta_{47}$ 温度计恢复古高度采用的样品主要为原生古土 壤中的钙质结核以及少量泥晶灰岩, 而古土壤钙质结 核在地质历史时期极易受到后期成岩改造的影响，使 得其原生沉积氧同位素组分发生改变, 因而影响古高 度重建的正确性，所以对原生古土壤结核的鉴别在古 高度重建中尤为重要. 前人在野外主要根据形态特征 判别古土壤，并使用放大镜选取没有明显重结晶特征 的钙质结核. 然后在室内将需要进行同位素分析的钙 质结核切片，利用XRD或者扫描电子显微镜鉴定样品 组分以及碳酸钙含量; 另外可通过显微镜观察样品的 微观形态特征, 选择没有明显重结晶, 无贯穿性方解石
脉体等后期成岩改造特征的结核作进一步的碳氧同位 素以及耦合同位素分析 ${ }^{[78]}$. 进行同位素分析的样品准 备一般利用微型牙钻, 在体视显微镜下钻取经过薄片 鉴定的结核粉末，所需样品量根据结核的碳酸钙含量 而定, 一次测量需要相当于纯碳酸钲 $6 \sim 10 \mathrm{mg}$ 样品. 其 次还可以通过古土壤上部砾岩中的灰岩砾石颗粒的碳 氧同位素特征检验样品是否经历了成岩改造 ${ }^{[10]}$. 如果 砾石颗粒的氧同位素特征与古土壤结核的氧同位素存 在明显区别, 则样品没有经历大规模的成岩改造作用.

随着碳酸盐耦合同位素的发展，作为温度指标的 碳酸盐耦合同位素温度计可以用于直接判定样品是 否经历了由温度升高引起的重结晶作用或者是固相 状态的同位素重排 ${ }^{[25,79]}$. 根据Quade等人 ${ }^{[25]}$ 对现今古 土壤结核的 $T\left(\Delta_{47}\right)$ 研究结果表明, 现今地表碳酸盐的 形成温度上限约为 $40^{\circ} \mathrm{C}$, 根据现今地温梯度推算古土 壤结核样品埋深应该不超过 $2.5 \sim 4 \mathrm{~km}$. 如果样品的埋 深大于 $2.5 \sim 4 \mathrm{~km}$, 重建的 $T\left(\Delta_{47}\right)$ 大于 $40^{\circ} \mathrm{C}$ 且与推测的 埋藏地温相当, 则样品很可能受到了埋深地温造成的 重结晶作用的改造; 若样品重建的 $T\left(\Delta_{47}\right)$ 大于 $100^{\circ} \mathrm{C}$ 且 与推测的埋藏地温不一致, 则样品可能发生了固相重 


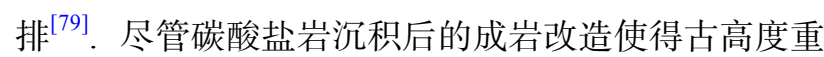
建的难度增加, 但是经受过成岩作用的样品的 $T\left(\Delta_{47}\right)$ 和 $\delta^{18} \mathrm{O}$ 由于 ${ }^{13} \mathrm{C}-{ }^{18} \mathrm{O}$ 结合键的重新排列可成为热成史 函数 ${ }^{[80 ~ 82]}$, 可应用于恢复原始沉积氧同位素值. 但是 现今的研究还未完全达到定量 $T\left(\Delta_{47}\right)$ 和 $\delta^{18} \mathrm{O}$ 的关系, 还需要对耦合同位素的同位素平衡交换机制进行深 人的研究 ${ }^{[79,83]}$.

\section{3 应用实例}

近年来使用 $\Delta_{47}$ 温度计对限制地球上最雄伟的山脉 和高原，如青藏高原、喜马拉雅山脉以及安第斯山脉 的隆升历史以及深部动力学机制提供了重要的约束. 下面就 $\Delta_{47}$ 温度计应用于南美洲的安第斯山脉，包括 Antiplano高原和Puna高原; 北美洲的科罗拉多高原, 落 基山脉和内华达地区; 青藏高原喜马拉雅地体的札达 盆地，拉萨地体的尼玛盆地、南木林盆地的以及林周 盆地古高度重建作重点阐释.

\subsection{1 南美洲安第斯中部}

位于安第斯山脉中部的Antiplano高原是一个宽约
$200 \mathrm{~km}$, 平均海拔在 $3800 \mathrm{~m}$ 的平坦高原, 其位于板块挤 压的褶皱冲断带东科迪勒拉山脉和活动的岩浆岛弧西 科迪勒拉山脉之间, 如图5(a).

对于南美安第斯山脉的古高度研究相对广泛，使 用的古高度重建方法有氧同位素古高度 ${ }^{[24]}$ 、古植物化 石古高度计 ${ }^{[84]}$ 以及 $\Delta_{47}$ 古高度计 ${ }^{[74 ~ 76,85]}$ ，如图5(a). Garzione等人 ${ }^{[24]}$ 使用传统氧同位素古高度计重建了Antiplano高原北部古高度，表明高原在11.4 10.3 Ma高度 在-700 $\pm 1000 \sim 1700 \pm 700 \mathrm{~m}$ 之间，7.6 6.8 Ma高度在 $1400 \pm 700 \sim 3400 \pm 500 \mathrm{~m}$ 之间，6.8 Ma以来高度在3500 $\pm 500 \sim 4200 \pm 500 \mathrm{~m}$ 之间(图5(b)). 上述传统氧同位素重 建古高度的误差达到上千米，这对于精确限制一个地 区的抬升历史并进一步限制其形成的地球动力学过程 是一个极大的挑战. 而Ghosh等人 ${ }^{[74]}$ 使用 $\Delta_{47}$ 温度计对 该地区相同样品重建古高度的结果表明, 11.4 10.3 Ma 高原高度为 $200 \pm 200 \mathrm{~m} ; 7.6 \sim 7.3 \mathrm{Ma}$ 高原高度为 $2500 \pm 500 \mathrm{~m} ; 6.7 \sim 5.8 \mathrm{Ma}$ 高原高度为3400 $\pm 500 \mathrm{~m}$ (图5 (b)), 上述结果使得古高度的误差从上千米减小到 $500 \mathrm{~m}$ 以下, 精确限制了地表的抬升速率仅为岩石圈增
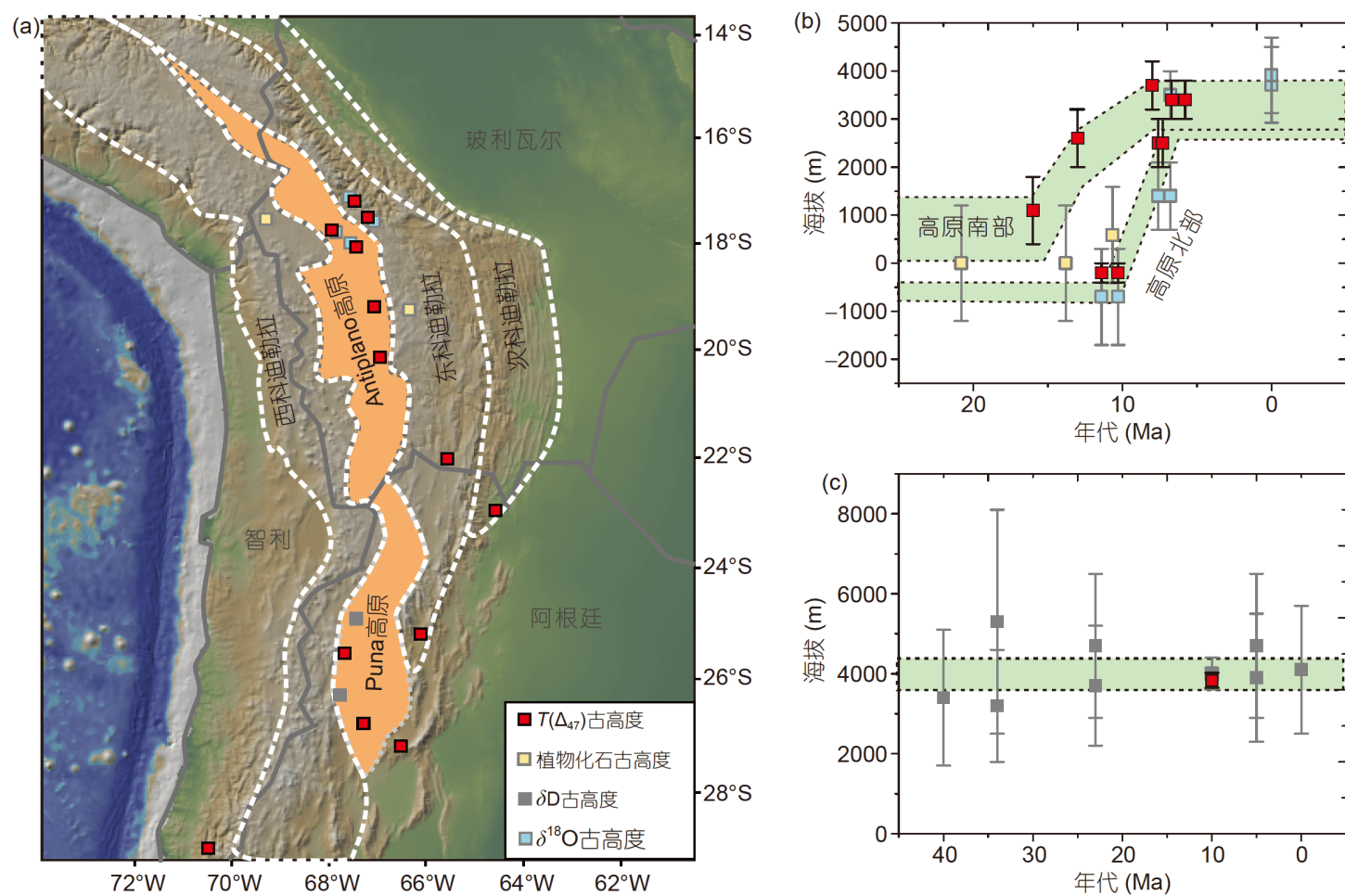

图 $5 \Delta_{47}$ 温度计在安第斯山脉的应用. (a) Antiplano高原和Puna高原 $\Delta_{47}$ 温度计应用于古高度研究点; (b) Antiplano高原古高度重建 ${ }^{[24,74,75,84]}$; (c) Puna高原古高度重建 ${ }^{[14,85]}$

Figure 5 Applications of $T\left(\Delta_{47}\right)$ in Andes. (a) Paleoelevation locations using $T\left(\Delta_{47}\right)$ within Antiplano and Puna Plateau; (b) paleoelevation reconstruction of Antiplano Plateau ${ }^{[24,74,75,84]}$; (c) paleoelevation reconstruction of Puna Plateau ${ }^{[14,85]}$ 
厚的 $1 / 3$, 由此得出岩石圈地幔拆沉为地表抬升的地球 动力学机制. 近来Garzione等人 ${ }^{[75]}$ 和Leier等人 ${ }^{[76]}$ 使用 $\Delta_{47}$ 温度计系统研究了Antiplano高原南部以及东科迪勒 拉的古高度, 结果表明Antiplano高原南部以及东科迪 勒拉的隆升和地幔拆沉时间发生在早中新世左右，比 高原北部早 $7 \pm 4 \mathrm{Ma}$ 左右, 造成上述差异的原因可能是 安第斯山脉中部地区地幔拆沉在时间和空间上的不一 致, 发生了片式拆沉.

位于Antiplano高原南部的Puna高原，与Antiplano 高原有着相似的构造背景. Canavan等人 ${ }^{[14]}$ 使用火山玻 璃氢同位素重建了Puna高原晚始新世-第四纪期间的 古高度，结果表明Puna高原自晚始新世便达到了现今 的海拔高度, 在中新世以来没有发生大规模的抬升. 该 研究结果与Carrapa等人 ${ }^{[85]}$ 使用 $\Delta_{47}$ 古温度计重建得到 的古高度结果相一致, 后者极大地缩小了古高度重建 的误差(图5(c)).

\subsection{2 北美洲中部}

$\Delta_{47}$ 温度计还应用于北美洲拉拉米造山带前陆系 统、Siever禇冲带、落基山脉中北部的山间盆地以及 华盛顿州的Chunstick盆地 ${ }^{[19,77,86-89]}$ (图6(a)).

科罗拉多高原位于科迪勒拉造山带的前陆，自显 生宙以来该地区保持着相对稳定的构造环境，地层未 遭受显著的变形, 与周边遭受显著变形的落基山脉, Seiver褶冲带形成鲜明对比. 玄武岩气孔古高度计研究 表明科罗拉多高原在晚第三纪期间显著抬升，抬升速 率达到 $220 \mathrm{~m} / \mathrm{Ma}^{[19]}$; 而低温热年代学研究表明南科罗
拉多高原在早第三纪期间就已经达到 $1500 \mathrm{~m}$ ，后期无 显著抬升 ${ }^{[90]}$. 为了解决两种古高度计重建结果之间存 在的矛盾, Huntington等人 ${ }^{[77]}$ 使用 $\Delta_{47}$ 古高度计研究了 科罗拉多高原新生代以来沉积的地层, 得出该地区自 中新世以来地表高度/温度梯度与现今相似，表明该地 区至少在晚中新世以来就达到了现今的海拔(图6(b)).

\subsection{3 青藏高原}

$\Delta_{47}$ 温度计在青藏高原古高度研究的应用主要分布 在喜马拉雅地体的札达盆地, 拉萨地体的尼玛盆地、 南木林盆地以及林周盆地 ${ }^{[73,91]}$ (图7(a)).

Saylor等人 ${ }^{[92]}$ 使用传统氧同位素对札达盆地的古 高度进行研究表明，盆地在中新世-上新世期间海拔高 度达到了 5300 5800 m左右，该结果与Wang等人 ${ }^{[18]}$ 使 用 $\Delta_{47}$ 古温度计对动物化石重建的古高度结果以及 Deng等人 ${ }^{[93]}$ 利用披毛犀和三趾马化石重建古高度结果 相当(图S3). 然而由于传统氧同位素温度计结果缺乏对 碳酸盐岩形成温度的准确限定，并且与札达盆地发现 的其他动物化石所显示的温暖湿润环境相悖 ${ }^{[94]}$, 使得 上述氧同位素和化石重建古高度结果受到质疑. 故 Huntington等人 ${ }^{[91]}$ 利用 $\Delta_{47}$ 温度计重新对札达盆地的古 土壤样品进行古高度重建, 结果表明札达盆地在晚中 新世-上新世期间的高度达到了 $5400 \mathrm{~m}(2 \sigma=500 \mathrm{~m})$ (图 S3), 后期由于东西向的伸展作用导致地表降低.

结合喜马拉雅地体的其他古高度研究结果 ${ }^{[3,73,95,96]}$ (图7(b)), 喜马拉雅地体在早始新世期间位于接近海平 面的位置, 晚始新世到中新世期间经历了快速抬升, 到
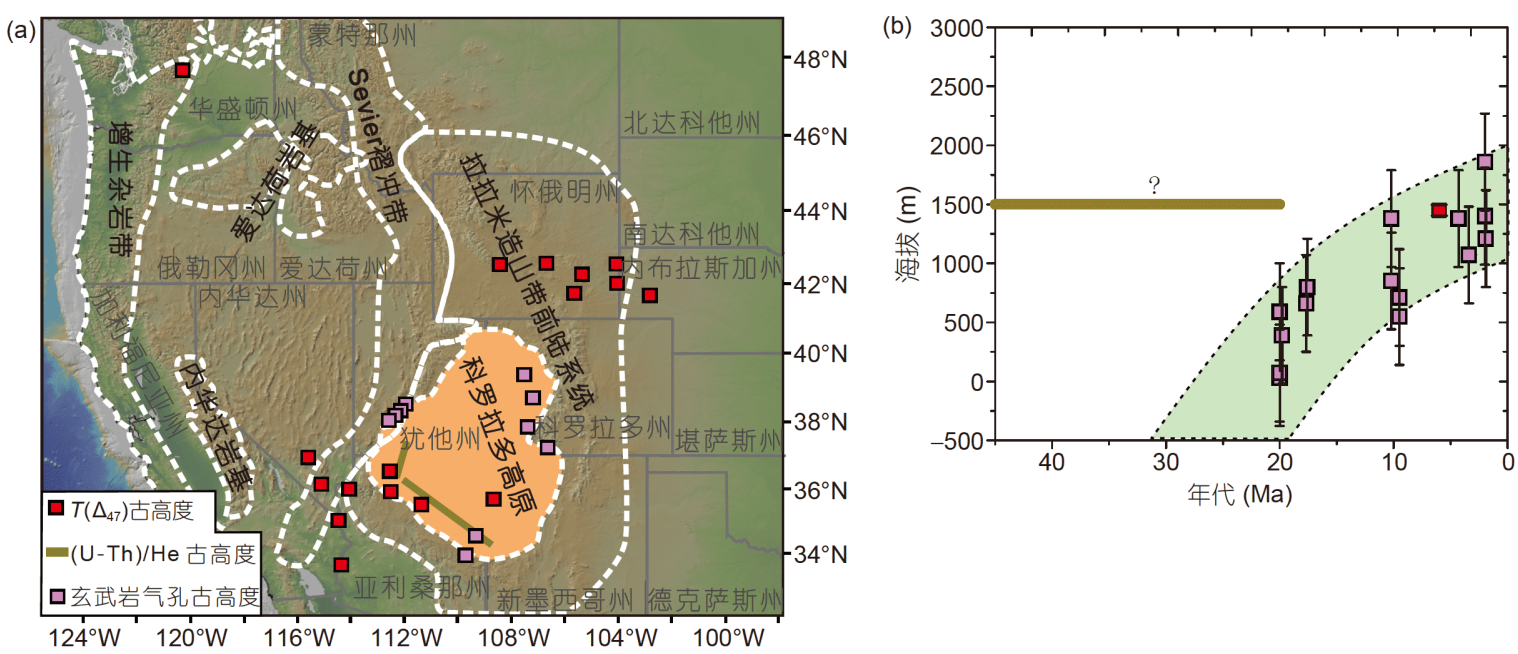

图 $6 \Delta_{47}$ 温度计在北美洲的应用. (a) 北美 $\Delta_{47}$ 温度计应用于古高度研究点; (b) 科罗拉多高原古高度重建 ${ }^{[77]}$

Figure 6 Applications of $T\left(\Delta_{47}\right)$ in North America. (a) Paleoelevation locations using $T\left(\Delta_{47}\right)$ in North America; (b) paleoelevation reconstruction of Colorado Plateau ${ }^{[77]}$ 

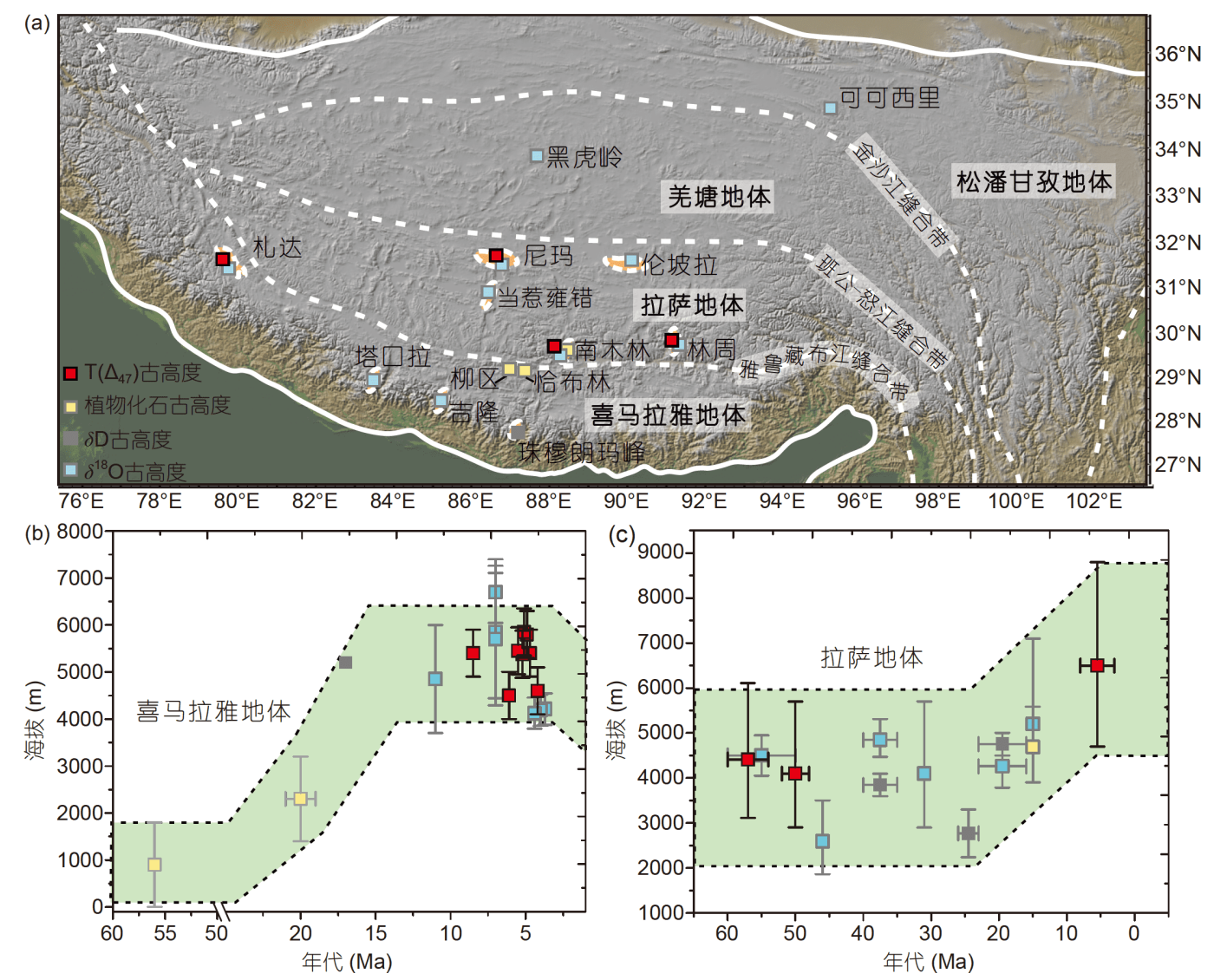

图 $7 \Delta_{4}$ 温度计在青藏高原的应用. (a) 喜马拉雅地体和拉萨地体 $\Delta_{47}$ 温度计应用于古高度研究点; (b) 喜马拉雅地体古高度重建 ${ }^{[3,73,91,95,96]}$; (c) 拉萨地体古高度重建 ${ }^{[2,15,23,73,97,98]}$

Figure 7 Applications of $T\left(\Delta_{47}\right)$ in Tibetan Plateau. (a) Paleoelevation locations using $T\left(\Delta_{47}\right)$ in Himalaya and Lhasa terranes; (b) paleoelevation reconstruction of Himalaya terrane $\mathrm{e}^{[3,73,91,95,96]}$; (c) paleoelevation reconstruction of Lhasa terrane ${ }^{[2,15,23,73,97,98]}$

晚中新世可能达到了高于现今的海拔，晚中新世之后 由于伸展垮塌导致海拔降低.

Huntington等人 ${ }^{[91]}$ 的研究还重新测量了尼玛盆地 样品的 $\Delta_{47}$ 值来重建其古地温, 结果显示尼玛盆地样品 的古地温都在 $40^{\circ} \mathrm{C}$ 以上, 判定样品经历了一定程度的 重结晶作用，表明以往使用古土壤结核重建的古高度 结果可能受到了后期成岩改造的影响.

Ingalls等人 ${ }^{[73]}$ 利用 $\Delta_{47}$ 温度计对南木林和林周盆地 的原生碳酸盐岩的研究表明，林周盆地在古新世期间 就抬升到了 $4000 \mathrm{~m}$ 以上，而南木林盆地至少在早渐新 世期间就达到了高海拔(6500 4100 m)(图7(c)). 该研究 结果与前人使用古植物化石和氧同位素的结果一

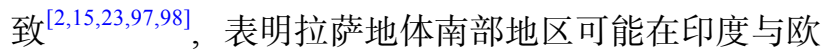
亚板块碰撞之前就已经抬升到高海拔，而拉萨地体北 部地区的抬升历史还有待进一步研究.

总结上述 $\Delta_{47}$ 温度计应用于古高度重建中的实例可
以看到, $\Delta_{47}$ 温度计作为热动力学温度计, 可以独立恢复 古地温，将其与已有的检验古土壤结核样品是否经历 成岩改造的手段相结合，可以准确地判定用于古高度 重建的样品是否保持其原始沉积时的同位素组分，由 此极大限度地提高了古高度重建的可信度. 基于 $\Delta_{47}$ 温 度计所能达到的误差极限 $\left( \pm 2^{\circ} \mathrm{C}\right)$, 使得古高度重建的 误差极大地缩小，进一步提高了古高度重建的精确性. 但是现今由于 $\Delta_{47}$ 温度计在同种矿物以及不同种类矿物 中的实验室转换方程之间都存在着差异性，造成使用 不同的转换方程所得到的 $T\left(\Delta_{47}\right)$ 差异较大，从而降低了 $\Delta_{47}$ 温度计的准确性，因此建立统一的实验室转换方程 尤为重要．另外由于碳酸盐岩结核样品本身容易受到 成岩改造作用而使得其同位素组分发生改变的特性, 使得应用 $\Delta_{47}$ 温度计重建古高度受到一定限制．对于这 些遭受成岩改造的样品, 如果能够通过 $T\left(\Delta_{47}\right)$ 和 $\delta^{18} \mathrm{O}$ 之 间存在的热成史函数定量的恢复原始沉积氧同位素值, 
则可在时间和空间尺度上扩大 $\Delta_{47}$ 温度计在古高度重建 中的应用范围.

\section{4 展望}

随着与 $\Delta_{47}$ 温度计相关的理论和实验方法的蓬勃发 展, 越来越多的实验室可以利用 $\Delta_{47}$ 温度计对古地温进 行精确的限制. 但 $\Delta_{47}$ 温度计依然存在许多亟待解决的 问题:（1）耦合同位素平衡交换反应的机制，各影响因 子对平衡反应的影响程度的量化 ${ }^{[83,99]}$; (2) 现今的合成 方解石 $\Delta_{47}$ 和温度转换方程整体上具有一致性，但是其 在低温范围下的差异依然未得到解决; 而生物成因碳 酸盐依旧没有统一的转换方程，需要对不同种类的生 物碳酸盐的平衡反应机制进行更多的研究; (3) 对于 $\Delta_{47}$ 温度计在除方解石碳酸盐岩之外含碳酸根岩石中的 应用需要更多研究和验证. 而对于 $\Delta_{47}$ 温度计在古高度 重建中的应用, 首先需要解决的是建立准确的碳酸盐 的 $\Delta_{47}$ 与温度的转换方程, 并且解决碳酸盐岩在经历后 期成岩改造之后，如何利用 $T\left(\Delta_{47}\right)$ 定量恢复其原始沉积 温度和同位素组分. 要解决这些问题, 今后的研究者需
要结合更多的理论基础，对于耦合同位素的热动力学, 晶体结构学原理作进一步的探讨; 在技术层面上通过 提高质谱仪的测量精度和更广泛的样品测试方面做出 更多的努力.

$\Delta_{47}$ 温度计能够独立而精确地恢复古地温，是一种 同时兼具准确度和精度的古温度计. 正是基于 $\Delta_{47}$ 温度 计的上述优点, 使其在构造重建特别是古高度重建中 得到了广泛应用，使得研究者可以更好地判定样品的 氧同位素值是否经历了成岩作用而发生变化，由此判 定样品用于古高度重建的可行性；同时对地貌的重建 也为重建该地区的抬升历史和解析其动力学机制提供 了精确可靠的指标. $\Delta_{47}$ 温度计在南美安第斯山脉的广 泛应用使得现今对于安第斯山脉的隆升历史和地球动 力学机制都有了更深人的理解. 但是在全球其他造山 带, 特别是对青藏高原隆升历史的研究还相对较少, 青 藏高原已有的古高度数据表明，青藏高原存在大量的 碳酸盐岩可以作为应用 $\Delta_{47}$ 温度计重建古高度的理想样 品，因此 $\Delta_{47}$ 温度计有望成为解决这一重大科学问题最 为有效的手段之一. 宝贵修改意见, 为进一步提高稿件的质量提供了必不可少的帮助.

\section{参考文献}

1 Garzione C N, Hoke G D, Libarkin J C, et al. Rise of the Andes. Science, 2008, 320: 1304-1307

2 Ding L, Xu Q, Yue Y, et al. The Andean-type Gangdese Mountains: Paleoelevation record from the Paleocene-Eocene Linzhou Basin. Earth Planet Sci Lett, 2014, 392: 250-264

3 Ding L, Spicer R A, Yang J, et al. Quantifying the rise of the Himalaya orogen and implications for the South Asian monsoon. Geology, 2017, 45: $215-218$

4 Husson L, Bernet M, Guillot S, et al. Dynamic ups and downs of the Himalaya. Geology, 2014, 42: 839-842

5 Flament N. Linking plate tectonics and mantle flow to earth's topography. Geology, 2014, 42: 927-928

6 Raymo M E, Ruddiman W F. Tectonic forcing of late Cenozoic climate. Nature, 1992, 359: 117-122

7 Zhisheng A, Kutzbach J E, Prell W L, et al. Evolution of Asian monsoons and phased uplift of the Himalaya-Tibetan Plateau since Late Miocene times. Nature, 2001, 411: 62-66

8 Molnar P, Boos W R, Battisti D S. Orographic controls on climate and paleoclimate of asia: Thermal and mechanical roles for the Tibetan Plateau. Annu Rev Earth Planet Sci, 2010, 38: 77-102

9 Rowley D B, Pierrehumbert R T, Currie B S. A new approach to stable isotope-based paleoaltimetry: Implications for paleoaltimetry and paleohypsometry of the High Himalaya since the Late Miocene. Earth Planet Sci Lett, 2001, 188: 253-268

10 DeCelles P G, Quade J, Kapp P, et al. High and dry in central Tibet during the Late Oligocene. Earth Planet Sci Lett, 2007, 253: 389-401

11 Xu Q, Ding L, Zhang L, et al. Paleogene high elevations in the Qiangtang Terrane, central Tibetan Plateau. Earth Planet Sci Lett, 2013, 362: 31-42

12 Jia G, Bai Y, Ma Y, et al. Paleoelevation of Tibetan Lunpola basin in the Oligocene-Miocene transition estimated from leaf wax lipid dual isotopes. Glob Planet Change, 2015, 126: 14-22

13 Hren M T, Pagani M, Erwin D M, et al. Biomarker reconstruction of the early Eocene paleotopography and paleoclimate of the northern Sierra Nevada. Geology, 2010, 38: 7-10

14 Canavan R R, Carrapa B, Clementz M T, et al. Early cenozoic uplift of the Puna Plateau, Central Andes, based on stable isotope paleoaltimetry of 
hydrated volcanic glass. Geology, 2014, 42: 447-450

15 Spicer R A, Harris N B W, Widdowson M, et al. Constant elevation of southern Tibet over the past 15 million years. Nature, 2003, 421: 622-624

16 Sun B, Wang Y F, Li C S, et al. Early Miocene elevation in northern Tibet estimated by palaeobotanical evidence. Sci Rep, 2015, 5: 10379

17 Deng T, Wang X, Fortelius M, et al. Out of Tibet: Pliocene Woolly Rhino suggests high-plateau origin of Ice Age Megaherbivores. Science, 2011, 333: $1285-1288$

18 Wang Y, Xu Y, Khawaja S, et al. Diet and environment of a mid-Pliocene fauna from southwestern himalaya: Paleo-elevation implications. Earth Planet Sci Lett, 2013, 376: 43-53

19 Sahagian D, Proussevitch A, Carlson W. Timing of Colorado Plateau uplift: Initial constraints from vesicular basalt-derived paleoelevations. Geology, 2002, 30: 807-810

20 Xia G Q, Yi H S, Zhao X X, et al. A late Mesozoic high plateau in eastern China: Evidence from basalt vesicular paleoaltimetry. Chin Sci Bull, 2012, 57: 2767-2777

21 Dupont-Nivet G, Hoorn C, Konert M. Tibetan uplift prior to the Eocene-Oligocene climate transition: Evidence from pollen analysis of the Xining basin. Geology, 2008, 36: 987-990

22 Song X Y, Spicer R A, Yang J, et al. Pollen evidence for an Eocene to Miocene elevation of central southern Tibet predating the rise of the High Himalaya. Palaeogeogr Palaeoclimatol Palaeoecol, 2010, 297: 159-168

23 Rowley D B, Currie B S. Palaeo-altimetry of the late Eocene to Miocene Lunpola basin, central Tibet. Nature, 2006, 439: 677-681

24 Garzione C N, Molnar P, Libarkin J C, et al. Rapid late Miocene rise of the Bolivian Altiplano: Evidence for removal of mantle lithosphere. Earth Planet Sci Lett, 2006, 241: 543-556

25 Quade J, Eiler J, Daëron M, et al. The clumped isotope geothermometer in soil and paleosol carbonate. Geochim Cosmochim Acta, 2013, 105: 92107

26 Gallagher T M, Sheldon N D. Combining soil water balance and clumped isotopes to understand the nature and timing of pedogenic carbonate formation. Chem Geol, 2016, 435: 79-91

27 Ma X F, Zhang Z F, Yan S, et al. An introduction to clumped isotope (in Chinese). J Earth Environ, 2012, 3: 950 -959 [马秀峰, 张兆峰, 严爽, 等. 耦合同位素简介. 地球环境学报, 2012, 3: 950-959]

28 Li P P, Ma Q Q, Zou H Y, et al. Basin principal of clumped isotopes and geological applications (in Chinese). J Palaeogeogr, 2017, 19: 713-728 [李平平, 马倩倩, 邹华耀, 等. 团簇同位素的基本原理与地质应用. 古地理学报, 2017, 19: 713-728]

29 Huntington K W, Lechler A R. Carbonate clumped isotope thermometry in continental tectonics. Tectonophysics, 2015, 647-648: 1-20

30 Eiler J M. "Clumped-isotope" geochemistry-The study of naturally-occurring, multiply-substituted isotopologues. Earth Planet Sci Lett, 2007, 262: $309-327$

31 Huntington K W, Eiler J M, Affek H P, et al. Methods and limitations of "clumped" $\mathrm{CO}_{2}$ isotope $\left(\Delta_{47}\right)$ analysis by gas-source isotope ratio mass spectrometry. J Mass Spectrom, 2009, 44: 1318-1329

32 Ghosh P, Adkins J, Affek H, et al. ${ }^{13} \mathrm{C}-{ }^{18} \mathrm{O}$ bonds in carbonate minerals: A new kind of paleothermometer. Geochim Cosmochim Acta, 2006, 70: $1439-1456$

33 Wang Z, Schauble E A, Eiler J M. Equilibrium thermodynamics of multiply substituted isotopologues of molecular gases. Geochim Cosmochim Acta, 2004, 68: 4779-4797

34 Guo W, Mosenfelder J L, Goddard III W A, et al. Isotopic fractionations associated with phosphoric acid digestion of carbonate minerals: Insights from first-principles theoretical modeling and clumped isotope measurements. Geochim Cosmochim Acta, 2009, 73: 7203-7225

35 Daëron M, Blamart D, Peral M, et al. Absolute isotopic abundance ratios and the accuracy of $\Delta_{47}$ measurements. Chem Geol, 2016, 442: 83-96

36 Schauer A J, Kelson J, Saenger C, et al. Choice of ${ }^{17} \mathrm{O}$ correction affects clumped isotope $\left(\Delta_{47}\right)$ values of $\mathrm{CO}_{2}$ measured with mass spectrometry. Rapid Commun Mass Spectrom, 2016, 30: 2607-2616

37 Eiler J M, Schauble E. ${ }^{18} \mathrm{O}^{13} \mathrm{C}^{16} \mathrm{O}$ in Earth's atmosphere. Geochim Cosmochim Acta, 2004, 68: 4767-4777

$38 \mathrm{He}$ B, Olack G A, Colman A S. Pressure baseline correction and high-precision $\mathrm{CO}_{2}$ clumped-isotope $\left(\Delta_{47}\right)$ measurements in bellows and microvolume modes. Rapid Commun Mass Spectrom, 2012, 26: 2837-2853

39 Bernasconi S M, Hu B, Wacker U, et al. Background effects on faraday collectors in gas-source mass spectrometry and implications for clumped isotope measurements. Rapid Commun Mass Spectrom, 2013, 27: 603-612

40 Dennis K J, Schrag D P. Clumped isotope thermometry of carbonatites as an indicator of diagenetic alteration. Geochim Cosmochim Acta, 2010, 74: $4110-4122$

41 Kluge T, John C M, Jourdan A L, et al. Laboratory calibration of the calcium carbonate clumped isotope thermometer in the $25-250^{\circ} \mathrm{C}$ temperature range. Geochim Cosmochim Acta, 2015, 157: 213-227

42 Kelson J R, Huntington K W, Schauer A J, et al. Toward a universal carbonate clumped isotope calibration: Diverse synthesis and preparatory methods suggest a single temperature relationship. Geochim Cosmochim Acta, 2017, 197: 104-131 
43 Zaarur S, Affek H P, Brandon M T. A revised calibration of the clumped isotope thermometer. Earth Planet Sci Lett, 2013, 382: 47-57

44 Came R E, Brand U, Affek H P. Clumped isotope signatures in modern brachiopod carbonate. Chem Geol, 2014, 377: 20-30

45 Eagle R A, Eiler J M, Tripati A K, et al. The influence of temperature and seawater carbonate saturation state on ${ }^{13} \mathrm{C}-{ }^{18} \mathrm{O}$ bond ordering in bivalve mollusks. Biogeosciences, 2013, 10: 4591-4606

46 Grauel A L, Schmid T W, Hu B, et al. Calibration and application of the "clumped isotope" thermometer to foraminifera for high-resolution climate reconstructions. Geochim Cosmochim Acta, 2013, 108: 125-140

47 Schmid T W, Bernasconi S M. An automated method for "clumped-isotope" measurements on small carbonate samples. Rapid Commun Mass Spectrom, 2010, 24: 1955-1963

48 Meckler A N, Ziegler M, Millán M I, et al. Long-term performance of the Kiel carbonate device with a new correction scheme for clumped isotope measurements. Rapid Commun Mass Spectrom, 2014, 28: 1705-1715

49 Müller I A, Fernandez A, Radke J, et al. Carbonate clumped isotope analyses with the long-integration dual-inlet (LIDI) workflow: Scratching at the lower sample weight boundaries. Rapid Commun Mass Spectrom, 2017, 31: 1057-1066

50 Wang X, Cui L, Li Y, et al. Determination of clumped isotopes in carbonate using isotope ratio mass spectrometry: Toward a systematic evaluation of a sample extraction method using a static porapak ${ }^{\mathrm{TM}} \mathrm{Q}$ absorbent trap. Int J Mass Spectrometry, 2016, 403: 8-14

51 Passey B H, Levin N E, Cerling T E, et al. High-temperature environments of human evolution in East Africa based on bond ordering in paleosol carbonates. Proc Natl Acad Sci USA, 2010, 107: 11245-11249

52 Petersen S V, Winkelstern I Z, Lohmann K C, et al. The effects of Porapak ${ }^{\mathrm{TM}}$ trap temperature on $\delta^{18} \mathrm{O}, \delta^{13} \mathrm{C}$, and $\Delta_{47}$ values in preparing samples for clumped isotope analysis. Rapid Commun Mass Spectrom, 2016, 30: 199-208

53 Dennis K J, Affek H P, Passey B H, et al. Defining an absolute reference frame for "clumped" isotope studies of $\mathrm{CO}_{2}$. Geochim Cosmochim Acta, 2011, 75: 7117-7131

54 John C M, Bowen D. Community software for challenging isotope analysis: First applications of "Easotope" to clumped isotopes. Rapid Commun Mass Spectrom, 2016, 30: 2285-2300

55 Bernasconi S M, Müller I A, Bergmann K D, et al. Reducing uncertainties in carbonate clumped isotope analysis through consistent carbonatebased standardization. Geochem Geophys Geosyst, 2018, 19: 2895-2914

56 Defliese W F, Hren M T, Lohmann K C. Compositional and temperature effects of phosphoric acid fractionation on $\Delta_{47}$ analysis and implications for discrepant calibrations. Chem Geol, 2015, 396: 51-60

57 Henkes G A, Passey B H, Wanamaker Jr A D, et al. Carbonate clumped isotope compositions of modern marine mollusk and brachiopod shells. Geochim Cosmochim Acta, 2013, 106: 307-325

58 Murray S T, Arienzo M M, Swart P K. Determining the $\Delta_{47}$ acid fractionation in dolomites. Geochim Cosmochim Acta, 2016, 174: 42-53

59 Spencer C, Kim S T. Carbonate clumped isotope paleothermometry: A review of recent advances in $\mathrm{CO}_{2}$ gas evolution, purification, measurement and standardization techniques. Geosci J, 2015, 19: 357-374

60 Tripati A K, Hill P S, Eagle R A, et al. Beyond temperature: Clumped isotope signatures in dissolved inorganic carbon species and the influence of solution chemistry on carbonate mineral composition. Geochim Cosmochim Acta, 2015, 166: 344-371

61 Hill P S, Tripati A K, Schauble E A. Theoretical constraints on the effects of $\mathrm{pH}$, salinity, and temperature on clumped isotope signatures of dissolved inorganic carbon species and precipitating carbonate minerals. Geochim Cosmochim Acta, 2014, 125: 610-652

62 Tang J, Dietzel M, Fernandez A, et al. Evaluation of kinetic effects on clumped isotope fractionation $\left(\Delta_{47}\right)$ during inorganic calcite precipitation. Geochim Cosmochim Acta, 2014, 134: 120-136

63 Ghosh P, Eiler J, Campana S E, et al. Calibration of the carbonate "clumped isotope" paleothermometer for otoliths. Geochim Cosmochim Acta, 2007, 71: 2736-2744

64 Tripati A K, Eagle R A, Thiagarajan N, et al. ${ }^{13} \mathrm{C}-{ }^{18} \mathrm{O}$ isotope signatures and "clumped isotope" thermometry in foraminifera and coccoliths. Geochim Cosmochim Acta, 2010, 74: 5697-5717

65 Thiagarajan N, Adkins J, Eiler J. Carbonate clumped isotope thermometry of deep-sea corals and implications for vital effects. Geochim Cosmochim Acta, 2011, 75: 4416-4425

66 Wacker U, Fiebig J, Tödter J, et al. Empirical calibration of the clumped isotope paleothermometer using calcites of various origins. Geochim Cosmochim Acta, 2014, 141: 127-144

67 Kimball J, Eagle R, Dunbar R. Carbonate "clumped" isotope signatures in aragonitic scleractinian and calcitic gorgonian deep-sea corals. Biogeosciences, 2016, 13: 6487-6505

68 Winkelstern I Z, Kaczmarek S E, Lohmann K C, et al. Calibration of dolomite clumped isotope thermometry. Chem Geol, 2016, 443: 32-38

69 Came R E, Azmy K, Tripati A, et al. Comparison of clumped isotope signatures of dolomite cements to fluid inclusion thermometry in the temperature range of $73-176^{\circ} \mathrm{C}$. Geochim Cosmochim Acta, 2017, 199: 31-47

70 Fernandez A, Tang J, Rosenheim B E. Siderite "clumped" isotope thermometry: A new paleoclimate proxy for humid continental environments. 
Geochim Cosmochim Acta, 2014, 126: 411-421

71 Daëron $\mathrm{M}$, Guo W, Eiler J, et al. ${ }^{13} \mathrm{C}^{18} \mathrm{O}$ clumping in speleothems: Observations from natural caves and precipitation experiments. Geochim Cosmochim Acta, 2011, 75: 3303-3317

72 Kele S, Breitenbach S F M, Capezzuoli E, et al. Temperature dependence of oxygen- and clumped isotope fractionation in carbonates: A study of travertines and tufas in the $6-95^{\circ} \mathrm{C}$ temperature range. Geochim Cosmochim Acta, 2015, 168: 172-192

73 Ingalls M, Rowley D, Olack G, et al. Paleocene to Pliocene low-latitude, high-elevation basins of southern Tibet: Implications for tectonic models of India-Asia collision, Cenozoic climate, and geochemical weathering. GSA Bull, 2017, 130: 307-330

74 Ghosh P, Garzione C N, Eiler J M. Rapid uplift of the altiplano revealed through ${ }^{13} \mathrm{C}-{ }^{18} \mathrm{O}$ bonds in paleosol carbonates. Science, 2006, 311: 511515

75 Garzione C N, Auerbach D J, Jin-Sook Smith J, et al. Clumped isotope evidence for diachronous surface cooling of the Altiplano and pulsed surface uplift of the Central Andes. Earth Planet Sci Lett, 2014, 393: 173-181

76 Leier A, McQuarrie N, Garzione C, et al. Stable isotope evidence for multiple pulses of rapid surface uplift in the Central Andes, Bolivia. Earth Planet Sci Lett, 2013, 371-372: 49-58

77 Huntington K W, Wernicke B P, Eiler J M. Influence of climate change and uplift on Colorado Plateau paleotemperatures from carbonate clumped isotope thermometry. Tectonics, 2010, 29: TC3005

78 Garzione C, Dettman D, Horton B. Carbonate oxygen isotope paleoaltimetry: Evaluating the effect of diagenesis on paleoelevation estimates for the Tibetan Plateau. Palaeogeogr Palaeoclimatol Palaeoecol, 2004, 212: 119-140

79 Henkes G A, Passey B H, Grossman E L, et al. Temperature limits for preservation of primary calcite clumped isotope paleotemperatures. Geochim Cosmochim Acta, 2014, 139: 362-382

80 Passey B H, Henkes G A. Carbonate clumped isotope bond reordering and geospeedometry. Earth Planet Sci Lett, 2012, 351-352: 223-236

81 Stolper D A, Eiler J M. Constraints on the formation and diagenesis of phosphorites using carbonate clumped isotopes. Geochim Cosmochim Acta, 2016, 181: 238-259

82 Xu Q C, Qiu N S, Liu W, et al. Reconstructing the basin thermal history with clumped isotope. Chin Sci Bull, 2019, 64: 556-578

83 Affek H P, Zaarur S. Kinetic isotope effect in $\mathrm{CO}_{2}$ degassing: Insight from clumped and oxygen isotopes in laboratory precipitation experiments. Geochim Cosmochim Acta, 2014, 143: 319-330

84 Gregory-Wodzicki K M. Uplift history of the Central and Northern Andes: A review. Geol Soc Am Bull, 2000, 112: 1091-1105

85 Carrapa B, Huntington K W, Clementz M, et al. Uplift of the Central Andes of NW Argentina associated with upper crustal shortening, revealed by multiproxy isotopic analyses. Tectonics, 2014, 33: 1039-1054

86 Lechler A R, Niemi N A, Hren M T, et al. Paleoelevation estimates for the northern and central proto-basin and range from carbonate clumped isotope thermometry. Tectonics, 2013, 32: 295-316

87 Fan M, Carrapa B. Late Cretaceous-Early Eocene Laramide uplift, exhumation, and basin subsidence in Wyoming: Crustal responses to flat slab subduction. Tectonics, 2014, 33: 509-529

88 Methner K, Fiebig J, Wacker U, et al. Eocene-Oligocene proto-Cascades topography revealed by clumped $\left(\Delta_{47}\right)$ and oxygen isotope $\left(\delta^{18} \mathrm{O}\right)$ geochemistry (Chumstick Basin, WA, USA). Tectonics, 2016, 35: 546-564

89 Snell K E, Koch P L, Druschke P, et al. High elevation of the "Nevadaplano" during the Late Cretaceous. Earth Planet Sci Lett, 2014, 386: 52-63

90 Flowers R M, Wernicke B P, Farley K A. Unroofing, incision, and uplift history of the southwestern Colorado Plateau from apatite (U-Th)/He thermochronometry. Geol Soc Am Bull, 2008, 120: 2225-2227

91 Huntington K W, Saylor J, Quade J, et al. High late Miocene-Pliocene elevation of the Zhada Basin, southwestern Tibetan Plateau, from carbonate clumped isotope thermometry. Geol Soc Am Bull, 2015, 127: 181-199

92 Saylor J E, Mora A, Horton B K, et al. Controls on the isotopic composition of surface water and precipitation in the Northern Andes, Colombian Eastern Cordillera. Geochim Cosmochim Acta, 2009, 73: 6999-7018

93 Deng T, Wang S Q, Xie G P, et al. A mammalian fossil from the Dingqing Formation in the Lunpola Basin, northern Tibet, and its relevance to age and paleo-altimetry. Chin Sci Bull, 2012, 57: 261-269

94 Wang X, Wang Y, Li Q, et al. Cenozoic vertebrate evolution and paleoenvironment in Tibetan Plateau: Progress and prospects. Gondwana Res, 2015, 27: 1335-1354

95 Garzione C N, Quade J, DeCelles P G, et al. Predicting paleoelevation of Tibet and the Himalaya from $\delta^{18} \mathrm{O}$ vs. Altitude gradients in meteoric water across the Nepal Himalaya. Earth Planet Sci Lett, 2000, 183: 215-229

96 Gebelin A, Mulch A, Teyssier C, et al. The Miocene elevation of Mount Everest. Geology, 2013, 41: 799-802

97 Polissar P J, Freeman K H, Rowley D B, et al. Paleoaltimetry of the Tibetan Plateau from D/H ratios of lipid biomarkers. Earth Planet Sci Lett, 2009, 287: 64-76

98 Xu Q, Ding L, Hetzel R, et al. Low elevation of the northern Lhasa terrane in the Eocene: Implications for relief development in south Tibet. Terra 
Nova, 2015, 27: 458-466

99 Affek H P. Clumped isotopic equilibrium and the rate of isotope exchange between $\mathrm{CO}_{2}$ and water. Am J Sci, 2013, 313: 309-325

\section{补充材料}

图S1 $\Delta_{47}$ 与 $T$ 理论计算

图S2 实验室合成方解石方法

图S3 扎达盆地古高度重建

本文以上补充材料见网络版csb.scichina.com. 补充材料为作者提供的原始数据, 作者对其学术质量和内容负责. 


\title{
Carbonate clumped isotope $\left(\Delta_{47}\right)$ thermometry and its application in paleoelevation reconstruction
}

\author{
Zhongyu Xiong ${ }^{1,3}$, Lin Ding ${ }^{1,2^{*}} \&$ Jing Xie ${ }^{1}$ \\ ${ }^{1}$ Key Laboratory of Continental Collision and Plateau Uplift, Institute of Tibetan Plateau Research, Chinese Academy of Sciences, Beijing 100101, \\ China; \\ ${ }^{2}$ Center for Excellence in Tibetan Plateau Earth Sciences, Chinese Academy of Sciences, Beijing 100101, China; \\ ${ }^{3}$ College of Resources and Environment, University of Chinese Academy of Sciences, Beijing 100049, China \\ * Corresponding author, E-mail: dinglin@itpcas.ac.cn
}

Paleoaltimetry provides crucial constraints on tectonic processes and precise paleotemperature measurement is an important component in paleoelevation reconstruction. Carbonate clumped isotope thermometry is based on homogeneous isotope equilibrium and is used to provide direct estimation of carbonate formation temperature independent of $\delta^{18} \mathrm{O}$ of the parent water from which the carbonate formed. The error regarding clumped isotope temperature estimation can be reduced to $\pm 2{ }^{\circ} \mathrm{C}$. The characteristics of temperature-dependence, and the small uncertainty using carbonate clumped isotope thermometry, greatly improve accuracy and precision in paleoelevation reconstruction.

In this review, we introduce the basic theory, laboratory methodology and standardization processes underpinning carbonate clumped isotope thermometry. Theoretical calculations indicate a linear relationship between parameter $\Delta_{47}$ and $1 / T^{2}$, which establishes the foundation from which carbonate clumped isotopes provide reliable paleotemperatures. The laboratory treatment of carbonate includes two systems: the modified Kiel IV device for small weight samples $(0.15-0.2 \mathrm{mg})$ installed within microvolume nitrogen traps and packed microvolume columns; and a heavy sample device that is installed with McCrea/common bath acid digestion devices, nitrogen traps and packed columns. These systems are designed to remove potential interference from water and hydrocarbons in the $\mathrm{CO}_{2}$. The measurement of purified $\mathrm{CO}_{2}$ is finished on gas source isotope mass ratio spectrometry (IMRS), especially on MAT 253 and 253 Plus machines. The standardization of the $\Delta_{47}$ to an absolute reference frame (ARF) accounts for the non-linearity and scaling effect of the IMRS, and the ARF also enables direct comparison of data from different laboratories. The practical procedure for the standardization includes interpolating the relationship between the measured $\delta_{47}$ and $\Delta_{47}$ of the equilibrium gases, and conversion of the measured $\Delta_{47}$ to theoretical values to derive an empirical transfer function.

Due to the great potential for solving Earth science problems, empirical calibration of the carbonate clumped isotope thermometry has been explored over a wide range of temperature in different types of carbonates, but is still plagued by discrepancies between different calibrations, probably due to variability in acid preparation, $\mathrm{CO}_{2}$ purification and data reduction methods. We summarized all the published synthetic calcite data to ARF with the same acid fractionation factor and yield a composite empirical calibration that could resolve the discrepancies between different calibrations. However, bio-genetic carbonates still fail to yield a universal empirical calibration, even when using the same methodology. The cause of this difference is probably due to different environmental, species discrimination and vital effects.

The application of carbonate clumped isotope thermometry to paleoaltimetry is based on the theory that the $\delta^{18} \mathrm{O}$ of precipitation (meteoric water) decreases proportionally as elevation increases. Knowing the formation temperature and $\delta^{18} \mathrm{O}$ of carbonate, we can trace back to the precise value of $\delta^{18} \mathrm{O}$ in precipitation, and then, using the theoretical model, or empirical relationship, between the $\delta^{18} \mathrm{O}$ of precipitation and elevation, reconstruct paleoelevation. Furthermore, clumped isotope thermometry can be used to test for diagenetic effects on pedogenic samples to ensure they retain original sedimentary isotopic compositions. We summarized previous applications of carbonate clumped isotope thermometry to accurately and precisely reconstruct the paleoelevation of the Andes mountains, Western North America and the Tibetan Plateau to provide insights into the uplift history and geodynamic process of those regions.

Despite the great potential for carbonate clumped isotopes in paleoelevation reconstruction, there are still challenges regarding equilibrium mechanisms, the discrepancies between different calibrations and the broader application in different carbonates. To resolve these challenges, more theoretical research is needed, as well as improving the resolution of IMRS.

carbonate clumped isotope, $\Delta_{47}$, paleothermometer, paleoelevation

doi: $10.1360 /$ N972019-00032 